\title{
A Unified Formation Control Scheme with a Single or Multiple Leaders
}

\author{
Nathan Sorensen and Wei Ren
}

\begin{abstract}
In the exploration and implementation of formation control schemes, bandwidth limitations and communication breakdown form a barrier to large scale formation control applications. The limitations of current formation control strategies involving a leader-follower approach and a consensusbased formation control approach with fully available group trajectory information are explored. A unified formation control scheme that accommodates an arbitrary number of group leaders and arbitrary information flow between vehicles is proposed. The scheme requires only local neighbor-to-neighbor information exchange. In particular, an extended consensus algorithm is applied on the group level to estimate the timevarying group trajectory information in a distributed manner. Based on the estimated group trajectory information, a consensus-based distributed formation control strategy is then applied for vehicle level control. The proposed scheme is experimentally implemented on a multi-robot platform under local neighbor-to-neighbor information exchange. The effect of a single or multiple leaders within the formation is also experimentally explored and discussed.
\end{abstract}

\section{INTRODUCTION}

In the field of cooperative control, algorithms for the achievement of formation maintenance among multiple vehicles have received significant attention. Given the limitations of communication bandwidth and communication range in many applications, the need for distributed algorithms that require only local neighbor-to-neighbor information exchange is apparent.

A typical leader-follower formation control strategy (e.g., [1]) assumes only one group leader within the team. In this case, only the group leader has the knowledge of group trajectory information, which is either preprogrammed in the group leader or provided to the group leader by an external source. The formation is then built on the reaction of the group to the motion of the group leader. The fact that only a single group leader is involved in the team implies that the leader-follower strategy is simple to implement and understand, and the requirement on communication bandwidth is reduced. This is, however, a single point massive failure type system because the loss of the group leader causes the entire group to fail. Another issue with the typical leaderfollower strategy is the lack of inter-vehicle information feedback throughout the group. For example, feedback from the followers is not used by the leader so the formation can become disjoint and followers can be lost if they are not able to track the motion of the leader accurately.

In order to overcome this type of single point failure tendency, much research has been focusing on in the areas

N. Sorensen and W. Ren are with the Department of Electrical and Computer Engineering, Utah State University, Logan, UT 84322, USA \{nwsorensen, wren\}@cc.usu.edu of decentralized or distributed cooperative control strategies where vehicle control laws are coupled and each vehicle makes its own decision according to the states of its neighbors. This allows the group to continue on to achieve an objective even in the presence of failure of any member of a group.

Among the distributed cooperative control strategies, consensus algorithms (see [2] for a survey) focus on driving the information states of all vehicles to a common value. For formation stabilization with a static formation centroid, if each vehicle in a group can reach consensus on the center point of the desired formation and specify a corresponding desired deviation from the center point, then vehicle formations can be achieved. To apply consensus algorithms to achieve formation maneuvering with a time-varying formation centroid trajectory, either the common formation velocity for the group or the desired group trajectory should be known by each vehicle in the group as in [3]-[6]. In particular, [5] assumes that a sequence of constant desired formation centroid states are preprogrammed on each vehicle. However, this approach cannot account for dynamically changing formation centroid states in response to dynamically changing situational awareness. While a flocking behavior can be achieved as in [7], [8] in the case that no vehicle has the knowledge of group formation velocity, a accurate formation geometry cannot be specified. In this paper, we focus on applications that require accurate formation geometry maintenance with desired group trajectory information involved.

The requirement that each vehicle have the knowledge of the desired group trajectory may not be realistic for many applications. For example, communication bandwidth and range limitations may prevent each vehicle in the group having access to the group trajectory information. Also, to increase stealth and flexibility, only a portion of the vehicles in the team may be provided with the desired group trajectory information. In addition, it is also possible that only a portion of the vehicles are able to detect a target or dangerous source at a certain time instant, and those vehicles in turn serve as the group leaders to guide the behaviors of the other group members.

Given the strength of the consensus algorithms for formation control with coupling involved between neighboring vehicles and the effectiveness of a traditional leader-follower approach when group trajectory information is limited in the formation, somehow integrating the two approaches yields the strength of both schemes. Bandwidth limitations for the group can be handled in limiting the amount of group trajectory information availability within the group, while robustness and greater group control is achieved with 
distributed nature of the consensus algorithms.

The main contribution of the current paper are twofold. First, we propose a unified distributed formation control scheme that accommodates an arbitrary number of group leaders and allows for arbitrary information flow between vehicles without adding complexity to the control law design and analysis. In particular, an extended consensus algorithm is applied on the group level to estimate the time-varying group trajectory information in a distributed manner. Based on the estimated group trajectory information, a consensusbased distributed formation control strategy is then applied for vehicle level control. Second, the proposed formation control scheme is experimentally implemented and validated on a multi-robot platform and the results are discussed. It is worthwhile to mention that although various strategies for decentralized or distributed formation control have been studied in the literature, few have been systematically verified on experimental platforms.

\section{A Unified Formation Control Scheme} by

Consider vehicles with single-integrator dynamics given

$$
\dot{r}_{i}=u_{i}, \quad i=1, \ldots, n
$$

where $r_{i}=\left[x_{i}, y_{i}\right]^{T}$ is the position and $u_{i}=\left[u_{x i}, u_{y i}\right]^{T}$ is the control input to the $i^{\text {th }}$ vehicle.

A consensus algorithm is given as

$$
u_{i}=-\sum_{j=1}^{n} g_{i j} k_{i j}\left(r_{i}-r_{j}\right), \quad i=1, \ldots, n,
$$

where $k_{i j}$ is a positive weighting factor, and $g_{i j}=1$ if information flows from vehicle $j$ to vehicle $i$ and 0 otherwise. The objective of (2) is to drive the information state of each vehicle toward the states of its local neighbors. For (2), consensus is reached asymptotically among the $n$ vehicles if $r_{i}(t) \rightarrow r_{j}(t), \forall i \neq j$, as $t \rightarrow \infty$ for all $r_{i}(0)$ (see [2] and references therein).

In this section, we propose a unified formation control scheme that accommodates an arbitrary number of group leaders and ensures accurate formation maintenance through information coupling between local neighbors.

One solution to formation control is the virtual structure approach (see [9] and references therein). The basic idea is to specify a virtual leader or a virtual coordinate frame located at the virtual center of the formation as a reference for the whole group such that each vehicle's desired states can be defined relative to the virtual leader or the virtual coordinate frame. As a result, single vehicle path planning and trajectory generation techniques can be employed for the virtual leader or the virtual coordinate frame while trajectory tracking strategies can be employed for each vehicle.

Fig. 1(a) shows an illustrative example of the virtual structure approach with a formation composed of four vehicles with planar motions, where $C_{o}$ represents the inertial frame and $C_{F}$ represents a virtual coordinate frame located at a virtual center $\left(x^{v c}, y^{v c}\right)$ with an orientation $\theta^{v c}$ relative to $C_{o}$. In Fig. 1(a), $r_{j}=\left[x_{j}, y_{j}\right]^{T}$ and $r_{j}^{d}=\left[x_{j}^{d}, y_{j}^{d}\right]^{T}$ represent, respectively, the $j^{\text {th }}$ vehicle's actual and desired position, and $r_{j F}=\left[x_{j F}, y_{j F}\right]^{T}$ represent the desired deviation of the $j^{\text {th }}$ vehicle relative to $C_{F}$, where $\left[\begin{array}{l}x_{j}^{d}(t) \\ y_{j}^{d}(t)\end{array}\right]=$ $\left[\begin{array}{l}x^{v c}(t) \\ y^{v c}(t)\end{array}\right]+\left[\begin{array}{cc}\cos \left(\theta^{v c}(t)\right) & -\sin \left(\theta^{v c}(t)\right) \\ \sin \left(\theta^{v c}(t)\right) & \cos \left(\theta^{v c}(t)\right)\end{array}\right]\left[\begin{array}{l}x_{j F}(t) \\ y_{j F}(t)\end{array}\right]$. If each vehicle can track its desired position accurately, then the desired formation shape can be preserved accurately.

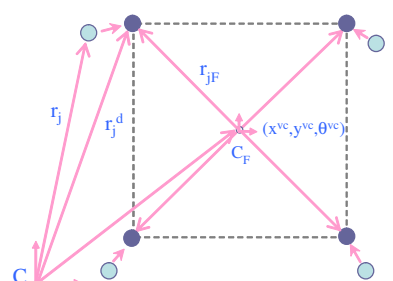

(a) A formation with a known virtual center.

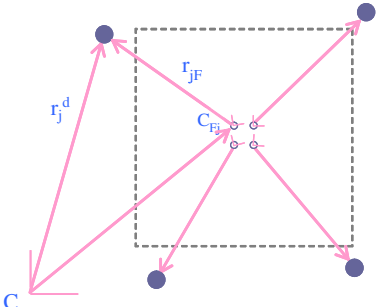

(b) A formation with inconsistent understanding of the formation center.
Fig. 1. A formation composed of four vehicles.

Note that Fig. 1(a) relies on the assumption that each vehicle knows the state of the virtual coordinate frame (i.e, virtual center and orientation), denoted as $\xi^{v c}=\left[x^{v c}, y^{v c}, \theta^{v c}\right]^{T}$, called formation state hereafter. However, this assumption is rather restrictive as described in Section I. In the case that each vehicle has inconsistent understanding or knowledge of $\xi^{v c}$ due to dynamically changing situational awareness or unreliable/limited communication, the formation geometry cannot be maintained as shown in Fig. 1(b), where $C_{F j}$ denotes the $j^{\text {th }}$ vehicle's understanding of the virtual coordinate frame.

Let $\xi_{i}^{v c}=\left[x_{i}^{v c}, y_{i}^{v c}, \theta_{i}^{v c}\right]^{T}$ denote the $i^{\text {th }}$ vehicle's understanding or estimation of the virtual coordinate frame. It is intuitive to apply the consensus algorithm (2) to guarantee that $\xi_{i}^{v c} \rightarrow \xi_{j}^{v c}$. However, this approach is only applicable for formation stabilization problems where the formation center and orientation are constant.

Next we propose a unified scheme for distributed formation control as shown in Fig. 2. The hierarchical architecture consists of three layers: consensus-based formation state estimation module, consensus-based formation control module, and physical vehicle. In Fig. $2, \mathcal{N}_{i}(t)$ and $\mathcal{J}_{i}(t)$ denote, respectively, the set of vehicles whose formation state estimates and position tracking error $r_{j}-r_{j}^{d}$ are available to vehicle $i$ at time $t$. The objective of the formation state estimation module is to drive $\xi_{i}^{v c}$ to $\xi_{d}^{v c}=\left[x_{d}^{v c}, y_{d}^{v c}, \theta_{d}^{v c}\right]^{T}$, which represents the desired state of the virtual coordinate frame available only to the group leaders. The local control law $u_{i}$ for each vehicle is based on its formation state estimate and the position tracking errors of its local neighbors.

On the formation state estimation level, each vehicle estimates the state of the virtual coordinate frame via an 


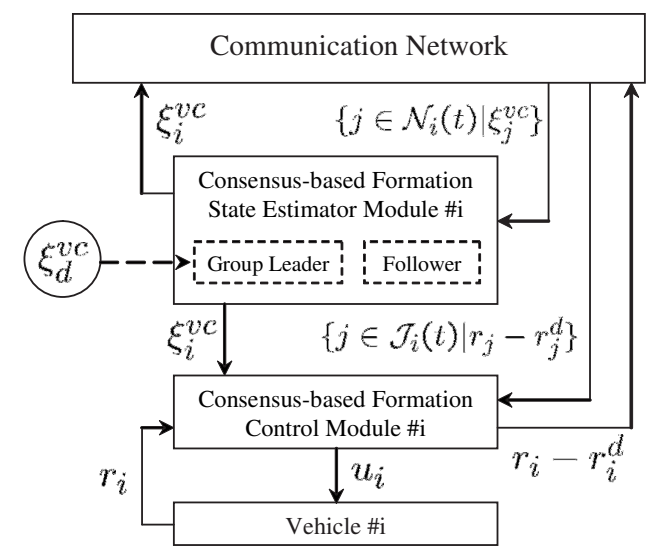

Fig. 2. A unified scheme for distributed formation control.

extended consensus algorithm as

$$
\begin{gathered}
\dot{\xi}_{i}^{v c}=\frac{\dot{\xi}_{d}^{v c}-\gamma\left(\xi_{i}^{v c}-\xi_{d}^{v c}\right)+\sum_{j=1}^{n} g_{i j}^{v c}\left[\dot{\xi}_{j}^{v c}-\gamma\left(\xi_{i}^{v c}-\xi_{j}^{v c}\right)\right]}{1+\sum_{j=1}^{n} g_{i j}^{v c}} \\
i \in \mathcal{L} \\
\dot{\xi}_{i}^{v c}=\frac{\sum_{j=1}^{n} g_{i j}^{v c}\left[\dot{\xi}_{j}^{v c}-\gamma\left(\xi_{i}^{v c}-\xi_{j}^{v c}\right)\right]}{\sum_{j=1}^{n} g_{i j}^{v c}}, \quad i \notin \mathcal{L}
\end{gathered}
$$

where $\mathcal{L}$ denotes the set of group leaders that have knowledge of $\xi_{d}^{v c}, g_{i j}^{v c}=1$ if vehicle $j$ 's estimate is available to vehicle $i$ and 0 otherwise, and $\gamma>0$. Note that only the group leaders have direct access to $\xi_{d}^{v c}$, which may be time varying, and the number of the group leaders can be any number from 1 to $n$.

Let $G^{v c}=\left[g_{i j}^{v c}\right] \in \mathbb{R}^{(n+1) \times(n+1)}$ be the adjacency matrix, where $g_{i j}^{v c}, \forall i, j \in\{1, \ldots, n\}$, are defined in (3), $g_{i(n+1)}^{v c}=1$ if $i \in \mathcal{L}$ and 0 otherwise, and $g_{(n+1) k}^{v c}=0$, $\forall k \in\{1, \ldots, n+1\}$. Then the estimation algorithm (3) guarantees that $\xi_{i}^{v c} \rightarrow \xi_{d}^{v c}$ asymptotically if and only if the directed graph of $G^{v c}$ has a directed spanning tree. The proof of the above argument is included in a companion paper [10]. Compared to (2), where consensus is reached on a constant value equal to the weighted average of the initial states, the estimation algorithm (3) reaches consensus a time-varying desired formation state. It is worthwhile to mention that [11], [12] also apply certain variants of consensus algorithms for formation control problems.

On the vehicle control level, we apply the following extended consensus algorithm as

$$
u_{i}=\dot{r}_{i}^{d}-\alpha_{i}\left(r_{i}-r_{i}^{d}\right)-\sum_{j=1}^{n} g_{i j} k_{i j}\left[\left(r_{i}-r_{i}^{d}\right)-\left(r_{j}-r_{j}^{d}\right)\right],
$$

where $\alpha_{i}>0, k_{i j}>0, g_{i j}=1$ if information flows from vehicle $j$ to vehicle $i$ and 0 otherwise, ${ }^{1}$ and $r_{i}^{d}=\left[x_{i}^{d}, y_{i}^{d}\right]^{T}$ with

$$
\left[\begin{array}{l}
x_{i}^{d} \\
y_{i}^{d}
\end{array}\right]=\left[\begin{array}{l}
x_{i}^{v c} \\
y_{i}^{v c}
\end{array}\right]+\left[\begin{array}{cc}
\cos \left(\theta_{i}^{v c}\right) & -\sin \left(\theta_{i}^{v c}\right) \\
\sin \left(\theta_{i}^{v c}\right) & \cos \left(\theta_{i}^{v c}\right)
\end{array}\right]\left[\begin{array}{l}
x_{i F} \\
y_{i F}
\end{array}\right]
$$

\footnotetext{
${ }^{1}$ Note that the estimation topology defined by $g_{i j}^{v c}$ may be different from the inter-vehicle information-exchange topology defined by $g_{i j}$.
}

With (4), (1) can be written in matrix form as $\dot{\tilde{r}}=$ $-\left[(L+\Gamma) \otimes I_{m}\right] \tilde{r}$, where $L$ is given as $\ell_{i i}=\sum_{j \neq i} g_{i j} k_{i j}$ and $\ell_{i j}=-g_{i j} k_{i j}, \forall i \neq j, \Gamma$ is a diagonal matrix with $\alpha_{i}$ being the diagonal entries, and $\tilde{r}=\left[\tilde{r}_{1}^{T}, \ldots, \tilde{r}_{n}^{T}\right]^{T}$ with $\tilde{r}_{i}=r_{i}-r_{i}^{d}$. Note that $-(L+\Gamma)$ is strictly diagonally dominant. From Gershgorin disc theorem [13], it is straightforward to see that all eigenvalues of $-(L+\Gamma)$ have negative real parts. Therefore, it follows that $\tilde{r} \rightarrow 0$ exponentially, that is, $r_{i} \rightarrow r_{i}^{d}, \forall i$. Under an arbitrary time-invariant informationexchange topology, the consensus algorithm (4) guarantees that $r_{i}(t) \rightarrow r_{i}^{d}(t), \forall i$, exponentially as $t \rightarrow \infty$. In other words, even a control law like $u_{i}=\dot{r}_{i}^{d}-\alpha\left(r_{i}-r_{i}^{d}\right)$ is sufficient to guarantee that $r_{i} \rightarrow r_{i}^{d}$. However, the coupling between neighboring vehicles denoted by the third term in (4) improves group robustness and reduces formation maintenance error.

Note that both (3) and (4) are distributed in the sense that only information exchange with local neighbors is required. The scheme in Fig. 2 accommodates an arbitrary number of group leaders and coupling in vehicle level control is achieved through information exchange with local neighbors. Approaches in [1], [5], [6] can be considered special cases of the scheme in Fig. 2. In particular, the approach in [1] corresponds to the case that only one group leader exists and each follower uses only the information from its unique parent node on the vehicle control level. The approach in [5] corresponds to the case that each vehicle behaves as a group leader and coupling on the vehicle control level occurs between one vehicle and its two adjacent neighbors. The approach in [6] corresponds to the case that each vehicle behaves as a group leader and coupling on the vehicle control level can occur between any local neighbors. The introduction of multiple group leaders neither complexify the control algorithms nor convergence analysis in (3) and (4). Multiple group leaders allow for reduction of a single point failure and allow time to connect to a new member of the group in case that one failure within the group occurs. The presence of an increased number of vehicles with accurate desired formation state information also tends to improve the formation state estimates of the other vehicles in practice as shown in Section III.

The desirable method for follower control is to let the followers know who the group leaders are. The group leaders may change throughout a formation motion, but they are expected to provide accurate information as to where the virtual coordinate frame should be. That is not to say that only one group leader is involved as in traditional single leader-follower control, but simply that in the estimation algorithm (3) all the vehicles that know the true formation state should be trusted rather than including opinions from vehicles that have no exact information of the true formation state. This type of enhanced knowledge follower is referred to as an intelligent follower hereafter.

In a dynamic environment, communication may be disrupted and the ability of the whole group to communicate with any specific member of a group cannot be ensured. General communication with as many members within the group 
as possible subject to communication bandwidth constraints is preferred to maintain a formation geometry. If a follower cannot communicate with a group leader due to limited communication range or other communication limitations, the follower can simply derive its formation state estimate based on information from the neighboring vehicles with whom it can communicate. This is a fail safe to ensure that the entire group is tied together as long as some type of weakly connected communication topology (i.e, existence of a directed spanning tree) exists within the formation. Of course, if information cannot be obtained from any member of the group or the communication topology becomes unconnected, the follower will fail. This group leader independent follower is referred to as a simple follower hereafter. The simple follower is a completely independent entity since it does not require the knowledge of who the group leader is to still maintain its position within the formation.

The application of group leaders, intelligent followers, and simple followers in an arbitrarily large group with an arbitrary weakly connected communication topology (i.e., the existence of a directed spanning tree) provides a basis for a unified approach to formation control. The distributed nature of this approach is robust to communication limitation and single point failure within the formation.

\section{EXPERIMENTAL Results OF Multi-Robot FORMATION CONTROL}

In this section, we experimentally implement the proposed formation control scheme on a multi-robot platform. We first conduct experiments with a single group leader and various numbers of intelligent and/or simple followers. We then conduct experiments involving two or three group leaders with various numbers of intelligent and/or simple followers to determine the effect of multiple group leaders within the group.

\section{A. Experimental Platform and Implementation}

An AmigoBot and P3DX based mobile robot platform as shown in Fig. 3 has been developed at Utah State University for the exploration of cooperative control strategies. The robots can communicate with each other through ethernet with TCP/IP protocols. The robots rely on encoder data for their position and orientation information.

In our experiments, we emulate limited inter-robot information exchange by simply disallowing the use of information obtained from certain members of the group although every robot can share information with every other robot. By doing so, we can test distributed cooperative control algorithms that involve only local neighbor-to-neighbor information exchange due to limited communication or sensing.

Let $\left(r_{x i}, r_{y i}\right), \theta_{i}$, and $\left(v_{i}, \omega_{i}\right)$ denote the Cartesian position, orientation, and linear and angular velocity of the $i^{\text {th }}$ robot respectively. The kinematic equations for the $i^{\text {th }}$ robot are

$$
\dot{r}_{x i}=v_{i} \cos \left(\theta_{i}\right), \quad \dot{r}_{y i}=v_{i} \sin \left(\theta_{i}\right), \quad \dot{\theta}_{i}=\omega_{i}
$$

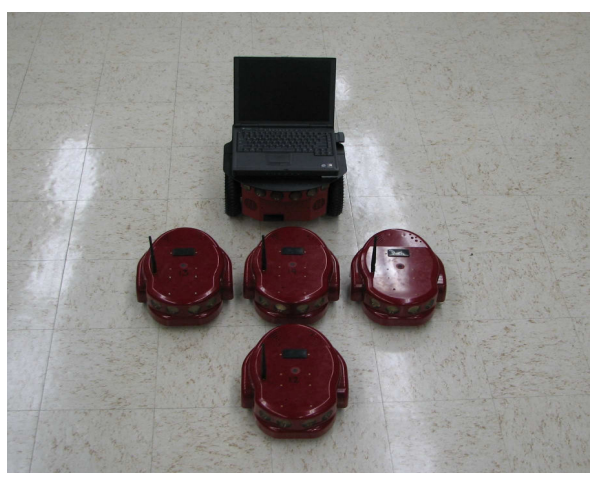

Fig. 3. AmigoBot and P3DX based mobile robot platform at USU.

One challenge to implementing the consensus algorithm (4) on our platform is that (4) requires single-integrator dynamics. To focus on the main issue, we feedback linearize (5) for a fixed point off the center of the wheel axis denoted as $\left(x_{i}, y_{i}\right)$, where $x_{i}=r_{x i}+d_{i} \cos \left(\theta_{i}\right)$ and $y_{i}=r_{y i}+d_{i} \sin \left(\theta_{i}\right)$ with $d_{i}=0.15 \mathrm{~m}$. Letting $\left[\begin{array}{c}v_{i} \\ \omega_{i}\end{array}\right]=\left[\begin{array}{cc}\cos \left(\theta_{i}\right) & \sin \left(\theta_{i}\right) \\ -\frac{1}{d_{i}} \sin \left(\theta_{i}\right) & \frac{1}{d_{i}} \cos \left(\theta_{i}\right)\end{array}\right]\left[\begin{array}{c}u_{x i} \\ u_{y i}\end{array}\right]$, gives $\left[\begin{array}{c}\dot{x}_{i} \\ \dot{y}_{i}\end{array}\right]=$ $\left[\begin{array}{l}u_{x i} \\ u_{y i}\end{array}\right]$, which is a simplified kinematic equation but is sufficient for the purpose of this paper.

In our experiments, a team of four AmigoBots are required to maintain a box formation, and the virtual coordinate frame follows a circle of $0.9 \mathrm{~m}$ radius with a linear speed of 0.05 $\mathrm{m} / \mathrm{sec}$ and angular speed of $0.03 \mathrm{rad} / \mathrm{sec}$. The virtual coordinate frame is initially located at $\left(x_{d}^{v c}(0), y_{d}^{v c}(0)\right)=(0,0)$ $\mathrm{m}$ with an orientation $\theta_{d}^{v c}=0 \mathrm{rad}$. Each robot applies (4) to derive $u_{x i}$ and $u_{y i}$ and (3) to estimate the formation state. In our experiments, we let $r_{j F}=\ell_{j}\left[\cos \left(\phi_{j}\right), \sin \left(\phi_{j}\right)\right]^{T}$ with $\ell_{j}=0.6 \mathrm{~m}$ and $\phi_{j}=\pi-\frac{\pi}{4} j \mathrm{rad}, j=1, \ldots, 4$.

\section{B. Formation Control with a Single Group Leader}

We first conduct a series of experiments employing a form of a single group leader with multiple followers to establish a basis for comparison with multi-leader based schemes.

In Case 1, we consider a single group leader with three intelligent followers. Fig. 4a shows the estimation topology, where a subscript $L$ denotes a group leader, a subscript $I$ denotes an intelligent follower, and a link from node $j$ to node $i$ denotes that $g_{i j}^{v c}=1$ in (3). Fig. 4b shows the inter-robot information-exchange topology, where a link from node $j$ to node $i$ denotes that $g_{i j}=1$ in (4).

Fig. 5 shows the experimental result in Case 1. In particular, Fig. 5a shows the trajectory of the four robots at $t \in\left[0, t_{f}\right] \mathrm{sec}$ and snapshots at $t=0, \frac{t_{f}}{3}, \frac{2 t_{f}}{3} \mathrm{sec}$, where $t_{f}$ is the ending time of the experiment. Fig. 5b shows the relative position error, defined as the difference between the desired and actual separation distance between the robots. Fig. 5c shows the virtual center position estimation error, defined as $\sqrt{\left(x_{d}^{v c}-x_{i}^{v c}\right)^{2}+\left(y_{d}^{v c}-y_{i}^{v c}\right)^{2}}$, where $\left(x_{d}^{v c}, y_{d}^{v c}\right)$ 
is the desired virtual center position known by the group leader. Fig. $5 \mathrm{~d}$ shows the virtual center orientation estimation error, defined as $\theta_{d}^{v c}-\theta_{i}^{v c}$, where $\theta_{d}^{v c}$ is the desired virtual center orientation known by the group leader. Note that the group is able to travel in tight formation around the circle with a formation error around $1 \mathrm{~cm}$ as shown in Fig. 5b. Also note that the initial virtual center estimation error converges in less than 2 seconds to an average value around $0.25 \mathrm{~cm}$, and the virtual center orientation estimation error is centered on zero. The asymptotic convergence is due to the asymptotic convergence of the consensus algorithm (3) used to estimate the formation state.

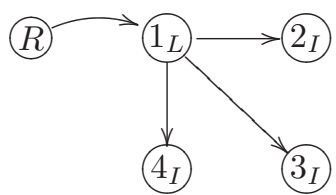

(a) Estimation Topology $g_{i j}^{v c}$

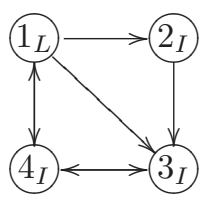

(b) Inter-robot Communication Topology $g_{i j}$
Fig. 4. Limited Graph Topologies in Case 1.

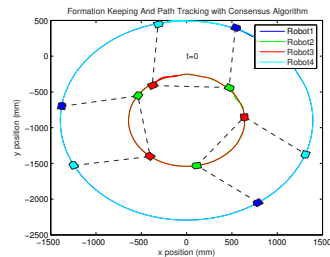

(a) Single Leader with Intelligent Followers Trajectory

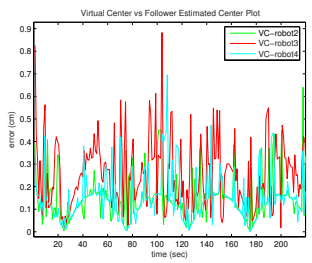

(c) Virtual Center Position Estimation Error

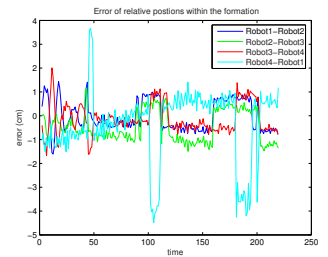

(b) Relative Position Error within Formation

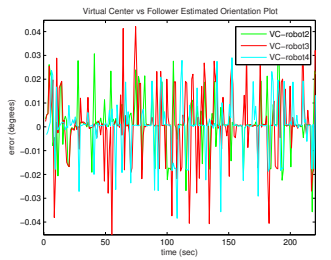

(d) Virtual Center Orientation Estimation Error
Fig. 5. Experimental results of a box formation with a single leader and three intelligent followers.

In Case 2, we consider a single group leader with three simple followers as shown in Fig. 6, where a subscript $S$ denotes a simple follower. Fig. 7 shows the experiential result in Case 2. Note that the average virtual center position estimation error is larger than in Case 1 due to the use of non-leader robots' estimations of the virtual center in the estimation algorithm. The average virtual center position estimation error is around $0.4 \mathrm{~cm}$ and the virtual center orientation estimation error is stationary around zero similar to Case 1.

In Case 3, we consider a group leader with one intelligent follower and two simple followers as shown in Fig. 8. The

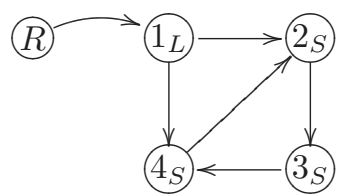

(a) Estimation Topology $g_{i j}^{v c}$

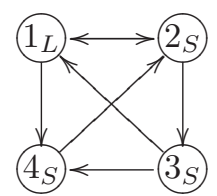

(b) Inter-robot Communication Topology $g_{i j}$
Fig. 6. Limited Graph Topologies in Case 2.

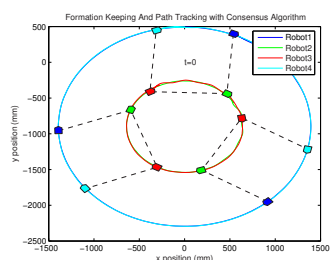

(a) Single Leader with Simple Followers Trajectory

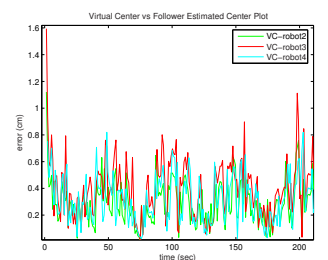

(c) Virtual Center Position Estimation Error

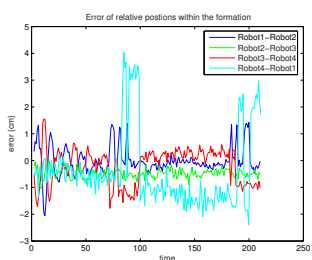

(b) Relative Position Error within Formation

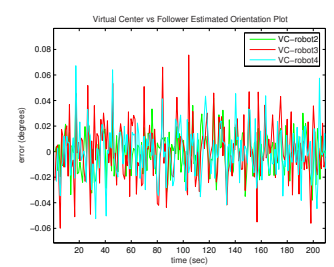

(d) Virtual Center Orientation Estimation Error
Fig. 7. Experimental results of a box formation with a single leader and simple followers.

experimental result in Case 3 is shown in Fig. 9. Given the inclusion of an intelligent follower in the group, the group leader provides a reduction in the virtual center estimation error for the simple followers and improves the overall group behavior. This is expected since the estimation error of the intelligent followers is much less than that of the simple followers. The greater number of better estimates included in the simple follower virtual center estimation algorithm, the more accurate the overall estimation will become.

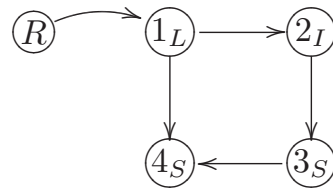

(a) Estimation Topology $g_{i j}^{v c}$

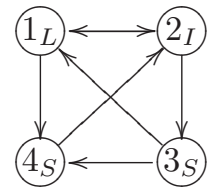

(b) Inter-robot Communication Topology $g_{i j}$
Fig. 8. Limited Graph Topologies in Case 3.

\section{Formation Control with Multiple Group Leaders}

In this section, multi-leader experiments are conducted to determine the overall effect of group leader placement and group leader number on the formation motion.

In Case 4, we consider two group leaders with two simple followers as shown in Fig. 10. The experimental result in 


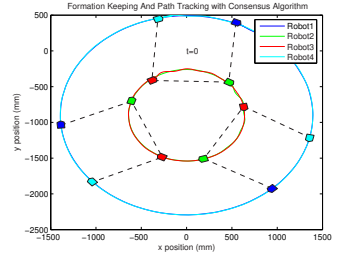

(a) Single Leader with a Intelligent and two Simple Followers Trajectory

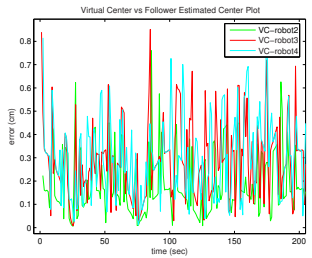

(c) Virtual Center Position Estimation Error

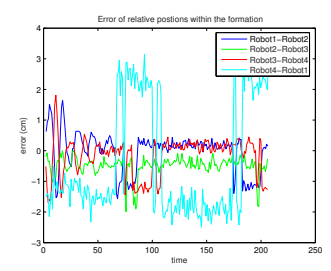

(b) Relative Position Error within Formation

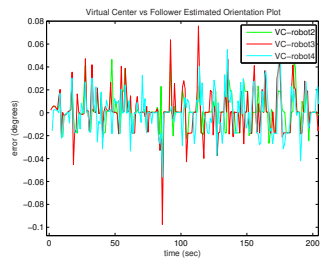

(d) Virtual Center Orientation Estimation Error
Fig. 9. Experimental results of a box formation with a single leader, an intelligent and two simple followers.

Case 4 is shown in Fig. 11, where it can be seen that the simple follower estimation error is reduced when the number of group leaders within the formation is increased compared to Cases 2 and 3. Although the follower robots do not know which robots in the formation are group leaders, the inclusion of the desired formation state information from the group leaders in the estimation algorithm for the follower robots allows the estimation to be much more accurate for both of the follower robots. This overall reduction of error is a direct effect of increasing the number of group leaders within the group.

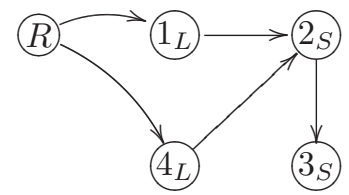

(a) Estimation Topology $g_{i j}^{v c}$

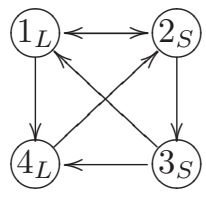

(b) Inter-robot Communication Topology $g_{i j}$
Fig. 10. Limited Graph Topologies in Case 4

In Case 5, we consider two group leaders with an intelligent follower and a simple follower as shown in Fig. 12. Compared to Case 4, the estimation error is reduced further and the overall formation is able to track tighter as shown in Fig. 13.

In Case 6, we consider three group leaders with a simple follower as shown in Fig. 14. As the number of group leaders is increased in the team, the formation error is further reduced as shown in Fig. 15.

Note that with the scheme in Fig. 2 all Cases 1-6 achieve good formation maintenance performance. We also notice that while in theory it follows that $\xi_{i}^{v c} \rightarrow \xi_{d}^{v c}, \forall i$, as long as the minimum connectivity (i.e., existence of a directed

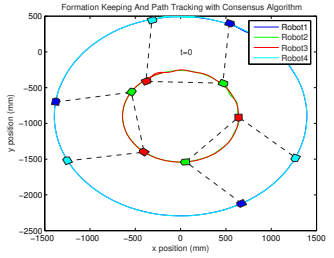

(a) Two Leaders with Two Simple Followers Trajectory

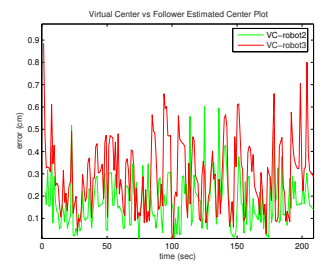

(c) Virtual Center Position Estimation Error

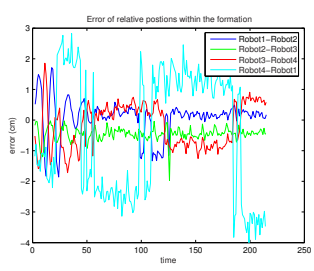

(b) Relative Position Error within Formation

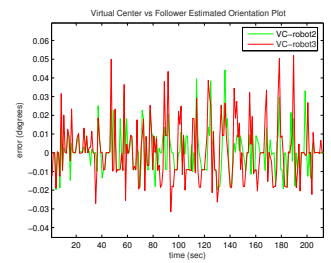

(d) Virtual Center Orientation Estimation Error
Fig. 11. Experimental results of a box formation with two leaders and two simple followers.

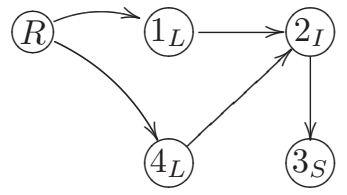

(a) Estimation Topology $g_{i j}^{v c}$

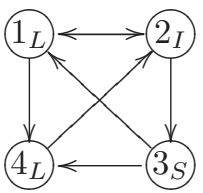

(b) Inter-robot Communication Topology $g_{i j}$
Fig. 12. Limited Graph Topologies in Case 5.

spanning tree) is ensured, we observe in our experiments that within a large formation, the more group leaders with which an intelligent follower can communicate the better the intelligent follower estimates. This then improves the estimation of the simple followers that cannot communicate with any group leader. The improvement in the performance in the presence of multiple group leaders is due to the real-time application of the consensus algorithm (3). The integration of the output from (3) introduces error into the estimation. The accumulated error is reduced at each sample time of the controller. The increase in the number of group leaders and intelligent followers in the group increases the weight in (3) toward values that are not suffering from integration error. With this increased weight, (3) increases the error dampening, which in turn reduces the overall error in the estimation and improves the overall formation performance. The greater the number of intelligent followers and group leaders, the better the formation tracking of the group trajectory.

\section{CONClusions And Future RESEARCH}

We have proposed a unified approach to formation control that accommodates an arbitrary number of group leaders and allows for arbitrary inter-robot coupling in the vehicle control law. By using an extended consensus-based estimation algorithm, the vehicles come into agreement on the 


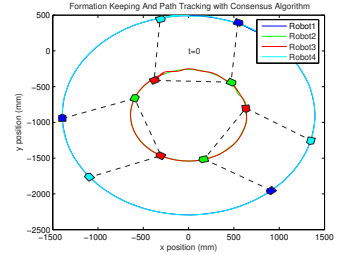

(a) Two Leaders with an Intelligent and a Simple Follower Trajectory

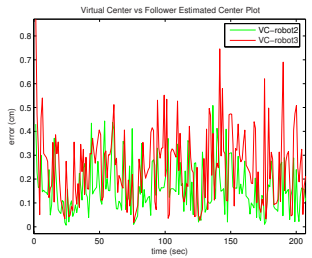

(c) Virtual Center Position Estimation Error

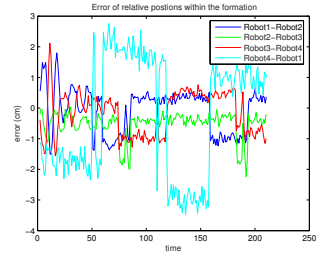

(b) Relative Position Error within Formation

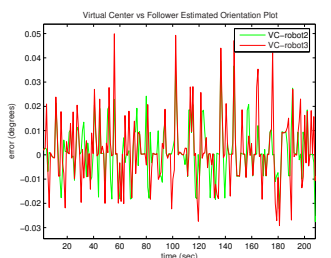

(d) Virtual Center Orientation Estimation Error
Fig. 13. Experimental results of a box formation with two leaders, an intelligent and a simple follower.

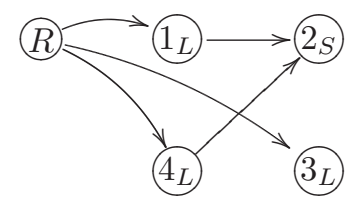

(a) Estimation Topology $g_{i j}^{v c}$

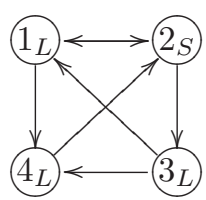

(b) Inter-robot Communication Topology $g_{i j}$
Fig. 14. Limited Graph Topologies in Case 6.

time-varying position and orientation of the virtual center. The vehicles then apply a consensus-based formation control algorithm to track their desired positions and preserve the formation geometry with their neighbors. The introduction of multiple group leaders and inter-robot coupling allows for individual leader and follower failure in the presence of limited communication and information within the formation. By increasing the number of group leaders within the formation, not only robustness against single point failure, but also vehicle estimate of the formation state is improved. The distributed nature of the proposed scheme does not require information as to which vehicles are group leaders, but this information can be used to improve the follower's formation state estimation when it is available. Experimental results on a multi-robot platform have shown the effectiveness of the scheme. An experimental demonstration of the proposed algorithms on a team of four AmigoBots can be found at http://www.engineering.usu.edu/ece/ faculty/wren/research.php. Future research will be to experimentally test the robustness of the scheme to group leader switching and inter-vehicle switching communication topologies. Research extending the experiments to an unmanned air vehicle (UAV) platform will also be conducted.

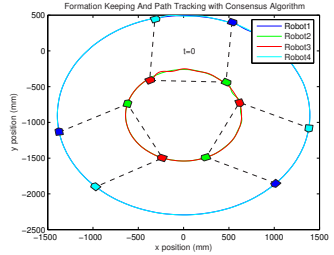

(a) Three Leaders with an Intelligent Follower Trajectory

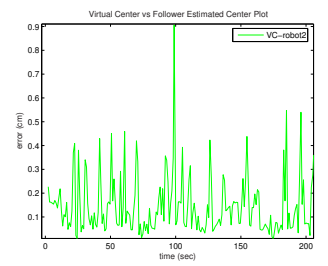

(c) Virtual Center Position Estimation Error

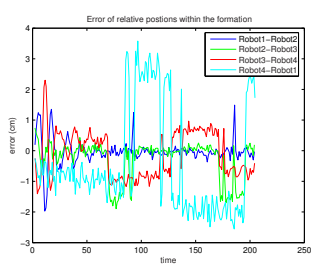

(b) Relative Position Error within Formation

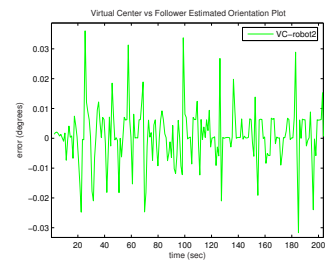

(d) Virtual Center Orientation Estimation Error
Fig. 15. Experimental results of a box formation with three leaders and an intelligent follower.

\section{REFERENCES}

[1] P. K. C. Wang, F. Y. Hadaegh, and K. Lau, "Synchronized formation rotation and attitude control of multiple free-flying spacecraft," AIAA J. of Guidance, Contr., and Dynamics, vol. 22, no. 1, pp. 28-35, January 1999.

[2] W. Ren, R. W. Beard, and E. M. Atkins, "Information consensus in multivehicle cooperative control: Collective group behavior through local interaction," IEEE Control Systems Magazine, April 2007.

[3] J. R. Lawton, R. W. Beard, and B. Young, "A decentralized approach to formation maneuvers," IEEE Trans. Robot. Automat., vol. 19, no. 6, pp. 933-941, December 2003.

[4] R. Olfati-Saber and R. M. Murray, "Flocking with obstacle avoidance: Cooperation with limited communication in mobile networks," in Proc. of Conf. on Decision Contr., Maui, Hawaii, December 2003, pp. 20222028.

[5] W. Ren and R. W. Beard, "Decentralized scheme for spacecraft formation flying via the virtual structure approach," AIAA J. of Guidance, Contr., and Dynamics, vol. 27, no. 1, pp. 73-82, January-February 2004.

[6] W. Ren, "Consensus based formation control strategies for multivehicle systems," in Proc. of American Contr. Conf., Minneapolis, MN, June 2006, pp. 4237-4242.

[7] C. W. Reynolds, "Flocks, herds, and schools: A distributed behavioral model," Computer Graphics, vol. 21, pp. 25-34, 1987.

[8] H. G. Tanner, A. Jadbabaie, and G. J. Pappas, "Stable flocking of mobile agents, part i: Fixed topology," in Proc. of Conf. on Decision Contr., Maui, Hawaii, December 2003, pp. 2010-2015.

[9] R. W. Beard, J. R. Lawton, and F. Y. Hadaegh, "A coordination architecture for spacecraft formation control," IEEE Trans. Contr. Syst. Technol., vol. 9, no. 6, pp. 777-790, November 2001.

[10] W. Ren, "Consensus seeking in multi-vehicle systems with a timevarying reference state," in Proc. of American Contr. Conf., New York, 2007.

[11] M. Porfiri, D. G. Roberson, and D. J. Stilwell, "Environmental tracking and formation control of a platoon of autonomous vehicles subject to limited communication," in Proc. of Conf. on Robot. Automat., Orlando, FL, May 2006, pp. 1050-4729.

[12] R. A. Freeman, P. Yang, and K. M. Lynch, "Distributed estimation and control of swarm formation statistics," in Proc. of American Contr. Conf., Minneapolis, MN, June 2006, pp. 749-755.

[13] R. A. Horn and C. R. Johnson, Matrix Analysis. Cambridge University Press, 1985. 